\title{
Helicase XPD Chaetomium thermophilum as a functional analogue of human XPD
}

Irina Petruseva

Institute of Chemical Biology and

Fundamental Medicine SB RAS

Novosibirsk, Russia

irapetru@niboch.nsc.ru

Natalia Lukyanchikova

Institute of Chemical Biology and

Fundamental Medicine SB RAS

Novosibirsk, Russia

lunata9@yandex.ru
Olga Lavrik

Institute of Chemical Biology and

Fundamental Medicine SB RAS

Novosibirsk, Russia

lavrik@niboch.nsc.ru

Jochen Kuper

Rudolf Virchow Center for

Experimental Medicine, University

Wurzburg, Germany

jochen.kuper@virchow.uniwuerzburg.de
Janette Kappenberger

Rudolf Virchow Center for

Experimental Medicine, University

Wurzburg, Germany

jeannette.kappenberger@uniwuerzburg.de

Rashid Anarbaev

Institute of Chemical Biology and

Fundamental Medicine SB RAS

Novosibirsk, Russia

anarbaev@niboch.nsc.ru

Caroline Kisker

Rudolf Virchow Center for

Experimental Medicine, University

Wurzburg, Germany

caroline.kisker@virchow.uniwuerzburg.de

\begin{abstract}
XPD helicase performs the verification step of damage recognition during NER. The detailed investigation of this step will help answer the question of interrelation of the chemical and spatial structure of the damage and its NER removal efficiency. The solution of this problem is also fundamentally important in the context of the induced cytotoxic DNA damages safekeeping and NER inhibitors search. Structurallyfunctional basis of the hXPD operatio as a damage verifier in NER $n$ in NER is not quite clear. Chaetomium thermophilum (ct) proteins have recently been proposed as more accessible and stable analogues of human ones. This study is a systematic analysis of the interaction of ctXPD and the more complete ctXPD-p44 system with DNA, which contain structurally different bulky lesions with previously estimated NER repair efficiencies. The data obtained indicate the similarity of the mechanisms for verifying damage by helicases ctXPD and $h X P D$ and prove the possibility of further use of ctXPD in extensive biochemical and structural studies of the mechanism for verifying damage.
\end{abstract}

Keywords - DNA repair, XPD helicase, bulky damages recognition/verification

\section{Motivation and Aim}

\section{Motivation}

The efficient functioning of DNA repair systems is a critical cell function, necessary to maintain genome stability. The efficiency of bulky damages elimination with nucleotide excision repair system (NER) is largely determined by the damage recognition efficiency. The key step of recognition damage verification with XPD and interrelation with the efficiency of damage eliminations is not sufficiently understood up to date.

Aim

The understanding of the mechanism and structure of $\mathrm{hXPD}$ is based on the results of biochemical studies, comparative mutagenesis, and studies of crystals of archaeal helicases which contain all domains necessary for XPD activities, however, operate as isolated monomers. A close Chaetomium thermophilum (ct)XPD functions as part of TFIIH available for isolation [1]. To prove the legitimacy of ctXPD as functional model of hXPD we have used the series of model DNA early characterized as NER substrates $[2,3]$.

\section{Methods}

XPD and auxillary ct protein p44 were expressed in bacterial system and isolated mainly as in [1]. To characterize ctXPD - damaged DNA interaction we have applied fluorescence spectroscopy and photoaffinity modification. CtXPD-damaged DNA affinity evaluation was based on fluorescence anisotropy measurements. The helicase activity was measured using the designed for like

DNA substrates: unmodified one and bearing exo-N-\{2[N(4-azido-2,5-difluoro-3-chloropyridin-6-yl)-

3aminopropionyl]aminoethyl -deoxycytidinine (FapdC); exoN-[4-(4-azido-2,3,5,6,-

tetrafluorobenzylidenehydrazinocarbonyl)-

butylcarbamoyl]- 2'-deoxycytidine (FabgdC); N-[6-(9antracenylcarbomoyl)hexanoyl]-3-amino-1,2-propandiol

(nAnt), N-[6-(5(6)-fluoresceinylcarbomoyl)hexanoyl]3amino-1,2-propandiol (nFlu) in translocated strand. When BHQ1 quencher was introduced in 3'-end of translocated strand, FAM group was at 5-end of the leaving strand; when at 3 '-end of translocated strand was $\mathrm{Cy} 3$ group, at 5 '-end of leaving stand was Dabcyl group. DNA probes for photocrosslinking were designed using photoactivable damages FapdC and FabgdC.

\section{Results}

The analysis of interaction of ctXPD with the set of the model DNAs revealed interconnection of the efficiency of NER performed elimination of structurally diverse bulky damages and their recognition with ctXPD. The increased ctXPD affinity for DNA bearing efficiently repairable damages (Fig.1) in row with the results of helicase activity measurements and XPD photocrosslinking results indicate the similarity of the mechanisms of damage verification by ctXPD and hXPD helicases. 


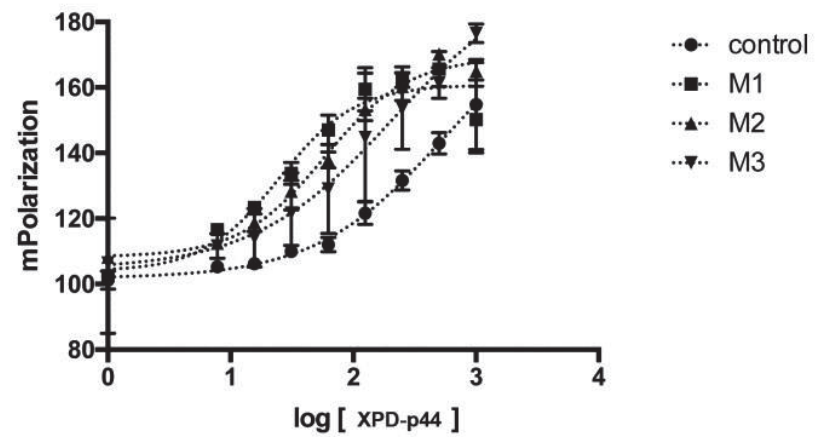

Fig. 1. XPD-p44 binding with DNA. Control - non modified DNA, M1 - nFlu-DNA; M2 - nAnt DNA; M3 - FapdC-DNA.
The data obtained in the given study prove legitimacy of ctXPD as the model for further investigations of bulky DNA damages verification during NER in higher eucaryotes and indicate correlation among efficiency of bulky damage elimination and helicase activity.

\section{ACKNOWLEDGMENT}

Supported by RFBR grant No. 19-04-00018.

\section{REFERENCES}

[1] Kuper J., Braun C., Elias A. et al. // pLoS Biol. 2014. V. 12 No. 9. p. 1-13.predictions and experiments. Progress in Biophysics and Molecular Biology. 107(1): 81-89)

[2] Evdokimov A., Petruseva I., Tsidulko A. et al. // Nucleic Acids Res. 2013. V. 41. No. 12. p. 1-10.Lashin S.A., Matushkin Yu.G. (2012) Haploid evolutionary constructor: new features and further challenges. In Silico. Biol. 11(3): 125-135.

[3] Evdokimov A., Tsidulko A., Popov A. et al. // DNA repair. 2018. V. 41. No. 61. p. 86-98. 